九州大学学術情報リポジトリ

Kyushu University Institutional Repository

\title{
Energy Efficient Distributed Coordination Function for IEEE 802.11 Wireless LAN
}

Adil, Bourichi

System LSI Research Centre, Graduate School of Information Science and Computer Engineering, Kyushu University

Tsuj imoto, Taizo

System LSI Research Centre, Graduate School of Information Science and Computer Engineering, Kyushu University

Yasuura, Hiroto

System LSI Research Centre, Graduate School of Information Science and Computer Engineering, Kyushu University

http://hdl. handle. net/2324/6264

出版情報: Proc. of IEEE Radio and Wireless Symposium 2006. 1，pp.323-326，2006-01. Institute of Electronics and Electrical Engineers

バージョン :

権利関係 : 


\title{
Energy Efficient Distributed Coordination Function for IEEE 802.11 Wireless LAN
}

\author{
Adil EL Bourichi, Taizo Tsujimoto, and Hiroto Yasuura \\ System LSI Research Centre, Graduate School of Information Science and Computer Engineering, \\ Kyushu University Fukuoka, 814-0001, Japan
}

\begin{abstract}
This paper proposes an approach to save the energy consumed by a computer's wireless network interface card (WNIC) due to the wireless communication activity in an IEEE 802.11 wireless local area network (WLAN). The energy efficiency design is considered at the MAC (Medium Access Control) layer, especially in its Distributed coordination Function (DCF), by reducing automatically the number of retransmissions due to collisions on the wireless channel.
\end{abstract}

Keywords Energy Efficiency, IEEE 802.11, MAC

protocol, CSMA/CA, RTS/CTS

\section{INTRODUCTION}

This paper addresses the problem of energy efficiency in wireless local area networks. Computers in a WLAN rely basically on batteries as their unique supply for electrical power. Because these batteries have a limited amount of energy, the rate at which this energy can be consumed in time, namely the power consumed, determines how long the battery will last. Energy efficient design aims at allowing the wireless NIC to operate at a low power, while not decreasing in performance, thus leading to less consumption of energy and therefore to a longer lifetime for the battery.

The studied WLAN in this paper is that of the IEEE 802.11 standard.

The goal of IEEE 802.11 standard [1] is to develop and maintain specifications for wireless connectivity for fixed, portable and moving stations within a local area. It provides MAC and physical layer (PHY) functionality.

IEEE 802.11 has two main configurations:

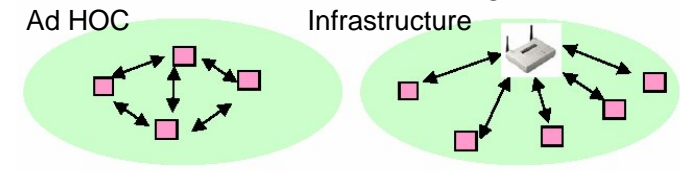

Fig. 1 Infrastructure and ad-hoc wireless LAN

1. Infrastructure: Nodes communicate with each other, and with an infrastructure wired network, through an Access Point (AP).
2. Independent (Ad-Hoc): Nodes communicate directly with each other, without an AP.

The Distributed Coordination Function (DCF) is the primary access protocol for the sharing of the wireless medium. DFC uses a carrier sense multiple access/collision avoidance (CSMA/CA) protocol, known also as the basic access scheme. Because of the possibility of partial network connectivity, wireless LAN protocols must take into account the hidden terminal problem (this occurs when a station is able to receive frames from two different stations but these two stations can not hear each other), see figure 2. To solve this, a four way handshaking mechanism is used through the exchange of two control frames: the Request to Send (RTS) and the Clear to Send (CTS) frames. RTS and CTS are normally shorter than the data frames. With RTS a station can for example reserve the channel for a certain duration of a transmission, the destination station approves by sending a CTS frame announcing this time to all stations that hear it, that way other stations will not attempt to access the medium during this time.

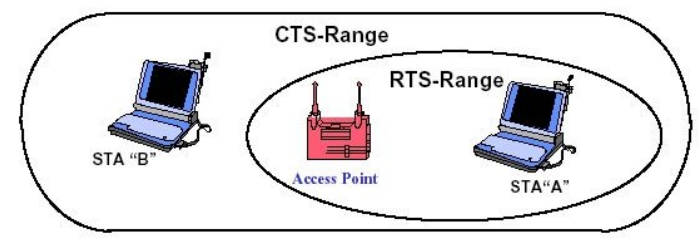

Fig. 2 Hidden Terminal Problem

When after transmitting a RTS no CTS is received the originating station can repeat the process faster than if a long data frame had been transmitted and corrupted, i.e. no ACK (Acknowledgement) frame had been detected.

RTS/CTS is optional, i.e. stations can choose to never use RTS/CTS or to use RTS/CTS whenever the data frame size exceeds a controllable parameter RTSThreshold. Previously proposed methods for energy efficiency in WLAN are presented in section 2. Section 3 gives the model used to estimate the energy consumed by a WNIC. Section 4 introduces the proposed method. Simulation and 
results are presented in Section 5. Section 6 concludes the paper.

\section{RELATED WORK}

IEEE 802.11 addresses energy efficiency by defining a Power Saving Mechanism (PSM) where stations are powered off (sleep mode) at periodic intervals [1]. E. Jung et al in [2] propose an energy-efficient PSM where nodes can dynamically adjust the size of their sleeping interval based on observed network conditions.

Reducing the WNIC's energy consumption can also be done at the physical level. For example by reducing the energy consumed by its RF parts (transmitter and receiver).

The receiver consumes energy when receiving packets and when sensing the channel. To conserve energy, IEEE 802.11 switches off the receiver periodically for nodes in PSM [1].

The transmitter consumes energy when transmitting packets or forwarding a packet in a multi-hop ad-hoc network. TPC (Transmit Power Control) methods aim at reducing the energy consumption by reducing the $\mathrm{RF}$ transmission power. But reduction of RF transmission power causes a higher bit error rate leading to collisions. The MAC reacts with retransmission of corrupted packets causing a higher power drain because of multiple retransmissions of the same packet. Increasing RF power results in higher channel reliability, therefore decreasing the bit error rate and the probability of retransmissions. But a higher RF power increases energy consumption. J.P. Ebert and A. Wolisz in [3] find that there is an optimum transmission power for a certain packet size and propose an algorithm where the MAC layer can tune to the appropriate power according to the packet size and channel characteristics.

\section{ESTIMATION OF ENERGY CONSUMPTION}

The radio can be in one state of the following: Transmitting, i.e. radio is transmitting data; Receiving, i.e. radio is effectively receiving data; Overhearing, i.e. radio is receiving data that is not destined to the node; Idle, i.e. radio is ready to receive or transmit; Sensing, i.e. radio has detected some signal but is not able to receive it; Sleeping, i.e. radio is in low power mode and thus is not able to receive or transmit. Sensing and overhearing are a special case of the receiving state. The radio can be in sleeping state only when the station is in the Power Saving Mode (PSM).

The next diagram summarizes the transition between these states:

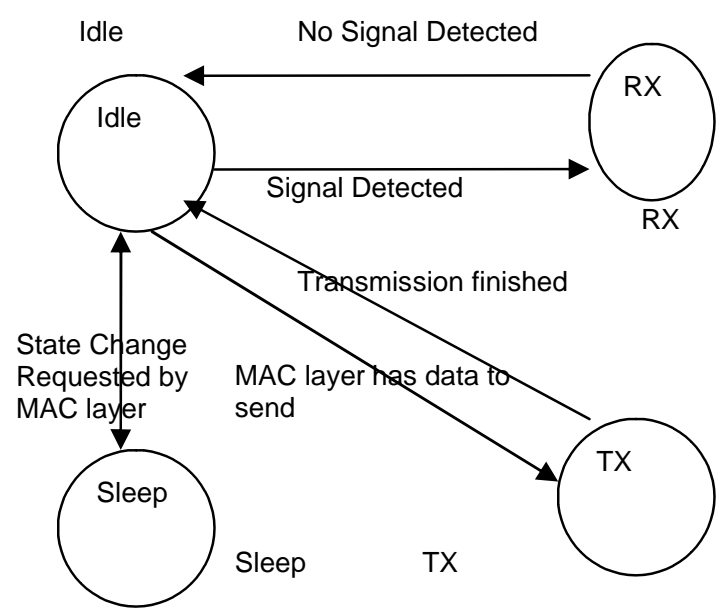

Fig. 3 MAC State Transition Diagram

For each state, the energy consumed is as $\mathrm{Ey}=\mathrm{Py} * \mathrm{Ty}$, where Py is the power dissipated by the radio while in state y, and Ty the time spent in state y. State y can be transmission, reception, idle, sleep or sensing.

The values of Py for each state $y$, used in the simulation, are given in section 5 .

\section{PROPOSED METHOD}

The main reason behind the high energy cost of the communication activity is the high number of retransmissions. These are due to an erroneous channel, interferences or collisions because of simultaneous transmissions. The first two causes of errors are not considered in this paper, frames are received in error only when they encounter collisions due to other simultaneous transmissions.

Follows that reducing the energy wasted in retransmissions would reduce the energy cost of the wireless communication activity and thus result in an energy saving for the overall system.

Therefore the goal of the method proposed in this paper is to reduce the energy wasted in retransmissions due to collisions occurring on the wireless medium. This can be achieved by limiting the number of retransmissions a data frame must endure before a successful transmission.

The method proposed in the paper opts for an orthodox way which is to not allow a second retransmission of a frame, while still competing to reserve the channel for transmitting the same frame. To do this, the paper proposes to implement the DCF such that it starts with the basic access scheme (no RTS/CTS) and when a collision happens, it switches to the RTS/CTS mechanism, stays in this mode until the frame has been successfully transmitted or the maximum number of allowed trials is reached, in 
which case the paper suggests to go back to the basic access scheme.

The reason for this choice is that, because the sizes of RTS and CTS control frames are much smaller than the data frames', retransmitting these control frames is less energy consuming than retransmitting the whole data frames.

\section{SIMULATION AND RESULTS}

The simulation is conducted in NS2 [4]. The energy consumed by a wireless ad-hoc network composed of $\mathrm{N}=$ 2, 3 ...30 nodes is calculated and compared in each of the three cases of:

1- Basic Scheme: ( No RTS/CTS used)

2- RTS/CTS: (RTS_Threshold is set to a default value of 512 bytes)

3- The Proposed Method: Automatically adjust RTS_Threshold to a minimal value after collisions (so as to activate RTS/CTS exchange ) and to a maximum value after every successful transmission of a frame (to go back to the basic access scheme)

The channel bit rate was set at $11 \mathrm{Mbps}$ and control frames were transmitted at the basic rate of $1 \mathrm{Mbps}$. The simulation time is 360 seconds and CBR (Constant Bit Rate) traffic is set between each pair of nodes in the network every 1 second, with all packets having the same size 1000 bytes.

The powers to transmit and to receive are 2.2 Watts and 1.35 Watts respectively, and no station is operating in the power save mode (PSM).

While it is important to evaluate the effect of the proposed method on the power consumption of the network, the power consumption alone is not enough to reflect the performance of the wireless LAN; for instance, if the network interface is constantly in a sleep mode, then the power consumption is at its lower level while actually no data is being transmitted. Therefore, we evaluate also the energy per good-put, defined as:

$E_{\text {bit_good }}[J / B i t]=\frac{\text { Average_Consumed_Power }[\mathrm{W}]}{\text { Goodput }[\text { Bit } / \mathrm{s}]}$.

The following two graphs draw respectively the average power consumption per node and the energy per goodput in function of the number of nodes in the network:

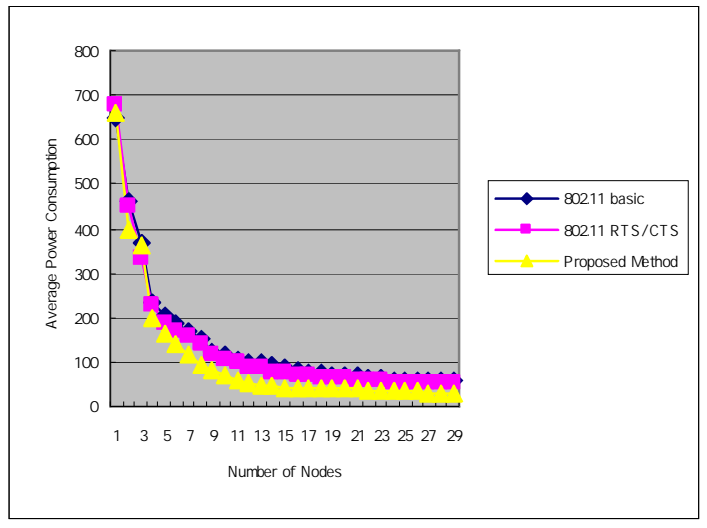

Fig.4 Average Power Consumption

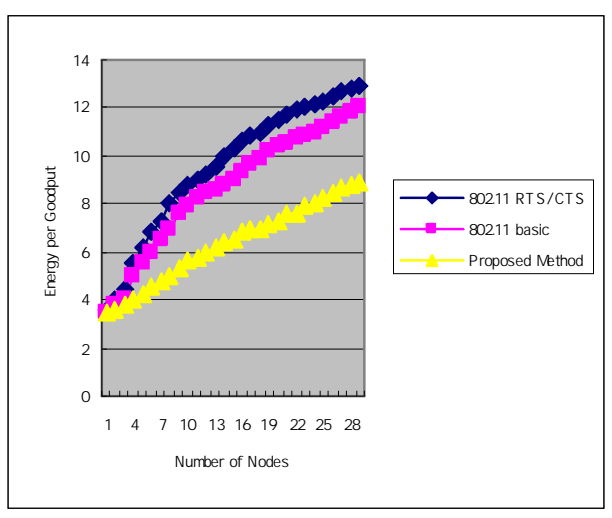

Fig.5 Energy per Goodput

\section{CONCLUSION}

The proposed method performs better than both the basic access and the RTS/CTS mode, with an energy saving of about $40 \%$ in average, especially for a number of nodes higher than 5 .

As a future work, the paper proposes to study the effect of an erroneous channel on the performance of the proposed method.

\section{ACKNOWLEDGEMENT}

This work has been supported by the Grant-in-Aid for the $21^{\text {st }}$ Century COE program. We are grateful for their contribution. 


\section{REFERENCES}

[1] IEEE 802.11 Working Group. "Part 11: Wireless LAN Medium Access Control (MAC) and Physical Layer (PHY) Specifications", ANSI/IEEE Std. 802.11 (1999)

[2] E. S. Jung et al., "Improving IEEE 802.11 Power Saving Mechanism," Texas A\&M University, Technical Report, July 2004.

[3] J-P. Ebert and A. Wolisz, "Combined Tuning of RF Power and Medium Access Control for WLANs", Mo MuC'99, , San Diego, USA, November 1999.

[4] NS2, www.isi.edu/nsnam/ns/ 